non-synonymous polymorphism in exon 4 of the Tapasin gene causing an arginine to threonine change that could affect tapasin function. We have investigated the effect of this SNP in resolving hepatitis C virus (HCV) infection in a cohort of 336 patients from the UK.

Aim We have investigated the effect of the SNP rs2071888 in resolving hepatitis C virus (HCV) infection in a cohort of 336 patients from the UK.

Method Genomic DNA from 216 chronically infected individuals and 120 spontaneous resolvers of HCV was genotyped with an allele specific PCR designed to identify the G/C SNP in exon 4 of the Tapasin gene. Individuals were also typed for HLA-A, -B and -C. Results were analysed for association with hepatitis $C$ outcome.

Results Overall the $\mathrm{G}$ allele of tapasin was associated with protection against chronic HCV infection ( $\mathrm{p}=0.018, \mathrm{OR}=1.99,95 \% \mathrm{CI}$ 1.14 to 3.46). Interestingly, in combination with heterozygosity at the HLA-B locus, heterozygosity at the tapasin locus was also protective (Abstract OP04 figure 1, $\mathrm{p}$ trend $=0.005$ ). Furthermore, we identified specific HLA-B alleles associated with protection in the context of the Tapasin G, but not Tapasin C allele. Specifically the G allele was most protective in the context of $B^{*} 0702(p=0.029$, $\mathrm{OR}=4.56,95 \%$ CI 1.2 to 17.27 ), and $\mathrm{B}^{*} 5701$ ( $\mathrm{p}=0.029, \mathrm{OR}=12,95 \%$ CI 1.2 to 120). Tapasin has been shown to bind specifically to amino acids 114 and 116 of HLA class I. Consistent with this functional interaction we found that aspartate at position 114 and serine at 116 were most beneficial in the context of both the G allele (D114/TapG, $\mathrm{p}<0.0001, \mathrm{OR}=3.3,95 \%$ CI 1.83 to 5.98; S116/TapG, $\mathrm{p}<0.0001$, $\mathrm{OR}=2.73,95 \%$ CI 1.57 to 4.76 ).

Conclusion The $\mathrm{G}$ allele of tapasin was associated with protection from chronic HCV infection, both alone and in combination with specific HLA class I alleles. Individuals who are heterozygous for both tapasin and HLA-B are relatively protected from chronic HCV infection. This heterozygosity may allow them to present a broader range of peptides to CTL and thus mount a more effective anti-HCV immune response.

\section{Transplant \\ OP05 THE IMPACT OF COMORBIDITIES ON OUTCOME OF PATIENTS ASSESSED FOR LIVER TRANSPLANTATION, WAITING LIST MORTALITY AND POST LIVER TRANSPLANTATION SURVIVAL USING THE CHARLSON COMORBIDITY INDEX}

doi:10.1136/gutjnl-2011-300857b.5

M A B Al-Freah, E Dionigi, L Blackmore, E Johnston, A Zubir, M Foxton, W Bernal, G Auzinger, M Rela, N D Heaton, J G O'Grady, M A Heneghan, J A Wendon. Institute of Liver Studies, King's College Hospital, London, UK

Introduction Severity of liver disease determines accurately the outcome of patients on liver transplant (LT) waiting list (WL). Comorbidities are known to affect post-LT outcomes but their effect on outcome of transplant assessment (TA) or WL mortality have not been fully explored in previous studies.

Aim To study the impact of comorbidities on TA, WL mortality and post LT survival.

Method Retrospective study of all patients assessed for LT at our centre between 1 January 2000 and 31 December $2007(n=1484)$. Patients with acute liver failure (175), amyloid (43), those assessed for re-LT (149) and 24 with incomplete information were also excluded. Nine comorbidities (Charlson Comorbidity Index - CCI) were prospectively defined according to Volk et al (Liver Transplant 2007;13:1515-20). Kaplan-Meier analysis was performed to determine impact of comorbidity on outcome. Cox regression hazard analysis was used to determine predictors of outcome and presented as (OR, 95\% CI, p value).

Results We analysed 1093 patients. Median age was 54 years (17-84), 67.5\% were men (738). There were 192 (17.6\%) patients with hepatocellular carcinoma (HCC). Patients with $\geq 1$ comorbidity were 499 (46.6\%) with most common comorbidities being diabetes $(23.2 \%)$ and renal dysfunction (12.1\%). Of 1093 assessed patients, $826(75.6 \%)$ were listed. Patients with $\geq 1$ comorbidity had significantly decreased LT free survival ( $\log$ rank $=33.586, p<0.001$ ). Multivariate analysis showed CCI $(1.79,1.52$ to $2.11, \mathrm{p}<0.001)$, age (1.03, 1.00 to $1.05, \mathrm{p}=0.035), \mathrm{Na}(0.93,0.89$ to $0.97, \mathrm{p}=0.001)$, MELD $(1.10,1.06$ to $1.14, \mathrm{p}<0.001)$ as being predictive. Of those listed for LT (826), 600 (72.6\%) were transplanted, 161 (19.5\%) died on WL and $65(7.9 \%)$ were delisted. Listed patients with $\geq 1$ comorbidity had significantly decreased LT free survival (log rank $=9.045, \mathrm{p}=0.003)$. Multivariate analysis showed CCI $(1.79,1.52$ to 2.11, $\mathrm{p}<0.001)$, age $(1.02,1.01$ to $1.03, \mathrm{p}=0.006)$, pre-LT Hb level (0.87, 0.80 to $0.95, p=0.003), \mathrm{Na}(0.96,0.93$ to $0.99, \mathrm{p}=0.014)$ and MELD $(1.14,1.11$ to $1.18, \mathrm{p}<0.001)$ were predictors of listing outcome. Transplanted patients with $\geq 1$ comorbidity had significantly decreased post LT survival (Log rank $=7.645, \mathrm{p}=0.006$ ). Multivariate analysis showed that only CCI $(1.36,1.13$ to 1.64 , $\mathrm{p}=0.001)$ and HCC (1.74, CI 1.21-2.51, $\mathrm{p}=0.003$ ) were independently associated with post-LT mortality. Similar post LT survival results seen when CCI was divided into 0,1 and $\geq 2$ comorbidities (Log rank $=11.342, \mathrm{p}=0.003$ ).

Conclusion We demonstrate that comorbidities significantly impact on the outcome of patients with chronic liver disease at TA, on wait-list and post LT survival. Adding CCI to known liver prognostic models may improve their prediction ability.

\section{Basic science OP06 MATERNAL OBESITY PROGRAMS OFFSPRING INNATE
IMMUNE DYSREGULATION IN NON-ALCOHOLIC FATTY LIVER DISEASE}

doi:10.1136/gutjnl-2011-300857b.6

${ }^{1}$ A Mouralidarane, ${ }^{1} \mathrm{~J}$ Soeda, ${ }^{2}$ Anne-Maj Samuelsson, ${ }^{2} \mathrm{P}$ Taylor, ${ }^{2} \mathrm{~L}$ Poston, ${ }^{1} \mathrm{~J}$ A Oben. ${ }^{1}$ University College London, UK; ${ }^{2}$ King's College London, UK

Introduction Obesity induced, non-alcoholic fatty liver disease (NAFLD), is the major cause of chronic liver dysfunction. In tandem, obesity and NAFLD rates in reproductive age women are rising. We have previously reported increased susceptibility to NAFLD in offspring exposed to maternal obesity via programming. Aim Our aim is to investigate the effects of maternal obesity on hepatic innate immune function in a more physiologically relevant model of NAFLD.

Method Female mice were fed standard or obesogenic chow, before, throughout pregnancy and in lactation. Offspring were then weaned onto either standard or obesogenic chow and studied at 3, 6 and 12 months. Read-outs included biochemical and histological indicators of NAFLD and fibrosis, hepatic triglycerides, gene expression analysis of pro-fibrotic pathways, FACS analysis of liver innate immune cells and flow cytometric detection of ROS.

Results Offspring only exposed to a post-weaning hyper-calorific diet (Group 2) exhibited raised leptin, ALT and hepatic triglyceride content compared to controls (Group 1) $(p<0.001)$. Moreover, hepatic gene expression of injury and fibrogenic markers were increased (Abstract OP06 table 1, $\mathrm{p}<0.01$ ). As expected, a more robust phenotype was observed at 12 compared to 3 months. Additionally, offspring exposed to maternal obesity plus a postweaning hyper-calorific diet (Group 3), displayed a more profound NAFLD phenotype with development of fibrosis and a NAFLD Activity Score $>5$. Mechanistically, we observed increased Kupffer cell numbers with impaired phagocytic function and raised ROS production, alongside reduced NKT cell numbers, in Group 3 compared to Group $1(\mathrm{p}<0.01)$. 